\title{
Value of the Frontal Assessment Battery Tool for Assessing the Frontal Lobe Function in Stroke Patients
}

\author{
Mihyang Han, MD ${ }^{1,2}$, Da-Ye Kim, $\mathrm{MD}^{1}$, Ja-Ho Leigh, $\mathrm{MD}^{2}$, Min-Wook Kim, MD, PhD ${ }^{1}$
}

\begin{abstract}
${ }^{1}$ Department of Rehabilitation Medicine, Incheon St. Mary's Hospital, College of Medicine, The Catholic University of Korea, Incheon; ${ }^{2}$ Department of Rehabilitation Medicine, Incheon Hospital of Korea Workers' Compensation and Welfare Service, Incheon, Korea
\end{abstract}

Objective To examine the correlation between the Frontal Assessment Battery (FAB) test, which is used to assess the frontal lobe function, and anatomical lesions as well as the ability of the test to detect frontal lobe dysfunction. Methods Records of stroke patients undergoing a FAB test and Mini-Mental State Examination (MMSE) were retrospectively reviewed. The patients were divided into three groups according to the lesions determined by an imaging study: frontal lobe cortex lesions, frontal subcortical circuit lesions, and other lesions. The FAB scores of the three groups were compared using the Kruskal-Wallis test. The validity of the FAB test to detect frontal lobe dysfunction was assessed by a comparison with the Computerized Neuropsychological Function Test (CNT) using the Spearman correlation coefficient. The correlation coefficients between the FAB test and MMSE were analyzed further based on the MMSE cutoff score.

Results Patients with frontal cortex lesions had significantly lower total and subtest scores according to the FAB test than the other patients. The FAB test correlated better with the CNT than the MMSE, particularly in the executive function and memory domains. A high MMSE score $(r=0.435)$ indicated a lower correlation with the FAB test score than a low MMSE score $(\mathrm{r}=0.714)$.

Conclusion The FAB test could differentiate frontal lobe lesions from others in stroke patients and showed a good correlation with the CNT. Moreover, the FAB test can be used in patients with high MMSE scores to detect frontal lobe dysfunction and determine the treatment strategies for stroke patients.

Keywords Stroke, Executive function, Neuropsychological tests, Mental status and dementia tests

\footnotetext{
Received August 2, 2019; Revised November 3, 2019; Accepted April 21, 2020; Published online July 28, 2020 Corresponding author: Min-Wook Kim

Department of Rehabilitation Medicine, Incheon St. Mary's Hospital, College of Medicine, The Catholic University of Korea, 56, Dongsu-ro, Bupyeonggu, Incheon 21431, Korea. Tel: +82-32-280-5207, Fax: +82-32-280-5040, E-mail: msdykim@catholic.ac.kr

ORCID: Mihyang Han (http://orcid.org/0000-0003-3869-9956); Da-Ye Kim (http://orcid.org/0000-0003-1831-6575); Ja-Ho Leigh (http://orcid. org/0000-0003-0465-6392); Min-Wook Kim (http://orcid.org/0000-0003-4505-809X).
}

@ This is an open-access article distributed under the terms of the Creative Commons Attribution Non-Commercial License (http://creativecommons.org/ licenses/by-nc/4.0) which permits unrestricted noncommercial use, distribution, and reproduction in any medium, provided the original work is properly cited. Copyright $\odot 2020$ by Korean Academy of Rehabilitation Medicine 


\section{INTRODUCTION}

The frontal lobe functions involve the executive function, attention, initiation, disinhibition, monitoring, language, and emotion control [1-3]. The frontal lobe cortex connects to other subcortical regions, including the striatum, basal ganglia, and thalamus. These connections are called the frontal subcortical networks and frontal subcortical circuits. Frontal subcortical circuits are balanced through direct and indirect pathways composed of neurotransmitters and receptors. The circuit plasticity is mediated by the striatal dopamine release following the cholinergic and gamma-aminobutyric acid agonist administration $[1,4]$. Many studies suggested that patients with lesions limited to the caudate nucleus, thalamus, and basal ganglia showed symptoms, such as those of frontal lobe syndrome caused by the frontal subcortical circuit destruction [4-6]. Parkinson disease, dementia, stroke, and alcohol abuse, which are characterized by frontal lobe dysfunction, are based on the neuropathology, including the frontal subcortical circuit.

Early detection of frontal dysfunction is needed to develop treatment strategies for diverse diseases [1]. Frontal lobe function assessment is clinically difficult, requiring extensive neuropsychological tests. In practice, these tests are time-consuming and difficult to apply to all patients because of the detailed options [7]. On the other hand, some brief screening tools focus on the frontal function, such as the Montreal Cognitive Assessment (MoCA), Severe Impairment Battery (SIB), and Addenbrooke's Cognitive Examination-Revised (ACE-R). The MoCA is a commonly used cognitive screening tool that is sensitive to executive functions. However, it is too difficult to perform test measurements for older patients, and it has floor effects. Most global cognitive screening tools, including the ACE-R and SIB, lack evaluations of some subdomains of the frontal function and are less sensitive to mild cognitive deficits [8].

The Frontal Assessment Battery (FAB) test, which consists of six subdomains of the frontal lobe, is a simple tool for the frontal lobe function assessment at the bedside [9]. The FAB test requires approximately 10 minutes to complete and is easy to administer. The reliability and validity of the FAB test have been proven in patients with a range of diseases. Further, the test showed good differentiation validity and reliability when used for those with
Parkinson disease [9-11]. In addition, it showed reliability for evaluations of frontal lobe dysfunction of patients with Alzheimer disease and those with frontotemporal dementia [12,13]. Cunha et al. [14] reported that the FAB test correlated with other neuropsychological tests of people with substance dependence. Paviour et al. [15] showed the FAB test validity for determining an executive dysfunction in patients with progressive supranuclear palsy.

One study evaluated the correlation between the FAB test and right hemispheric lateral frontal lobe dysfunction in stroke patients. The FAB test showed good validity in the detection of regional dysfunction [16]. Although stroke is a prevalent problem that affects the function of the frontal lobe, few studies have examined the usefulness of the FAB test for stroke patients compared to other neuropsychological tests. Furthermore, no studies have evaluated the FAB test for differentiating lesions of the frontal subcortical circuit from other types of lesions in stroke patients.

This study evaluated the usefulness of FAB test in differentiating anatomical lesions according to the degree to which the frontal subcortical circuit is involved as well as the validity of FAB test to detect frontal lobe dysfunction by comparing with the Computerized Neuropsychological Function Test (CNT). This study also compared the FAB test with the Mini-Mental State Examination (MMSE), which is one of the most frequently used tools for evaluating cognition in general. In addition, this study evaluated whether the FAB test is more valuable than the MMSE in terms of the value in frontal lobe dysfunction detection.

\section{MATERIALS AND METHODS}

\section{Subjects}

Records of patients who were admitted to the Departments of Neurology, Neurosurgery, and Rehabilitation Medicine at a tertiary referral brain center for the treatment of stroke and related symptoms from November 2016 to December 2017 were retrospectively obtained. Patients who were evaluated for their cognitive function using the MMSE and FAB tools were enrolled in this study. Those with other causes of frontal lobe dysfunction, such as hypoxic brain injury, traumatic brain injury, Parkinson disease, brain tumors, and previous neuro- 
logic disorders, were excluded.

The patients were divided into the following three groups based on the magnetic resonance imaging or computed tomography findings: (1) FL group, with lesions involving the frontal lobe cortex; (2) SC group, with lesions related to the frontal subcortical circuit without frontal cortex involvement; and (3) OL group, with other lesions that were unrelated to the frontal subcortical circuit. Data collected included the age at stroke onset, sex, type of stroke, side of stroke, Modified Barthel Index (MBI), and initial National Institutes of Health Stroke Scale (NIHSS) score. The use of patient data for research purposes was approved by the research ethics committee at the Incheon St. Mary's Hospital, Catholic University of Korea (Approval No. OC18RESI0006).

\section{Neuropsychological evaluation}

All patients were administered with the MMSE to assess their general cognitive function and the FAB test to detect any impairment in the frontal lobe function. Within the MMSE, the subtest for the executive function was a three-step command test $[17,18]$. The subtest related to a frontal lobe function, including attention and repetition, was analyzed further. The FAB test consisted of the following six subtests: (1) questions about the similarities between two objects to evaluate conceptualization and abstract thinking; (2) questions asking the participant to list as many words as possible starting with a specific letter within 1 minutes to assess their mental flexibility and verbal fluency; (3) questions asking individuals to perform the Luria manoeuvre and fist-edge-palm patterns to determine if their programming and motor acts are correctly executed; (4) questions requesting the participants to provide an opposite response to the examiner's signals (conflicting instructions) to assess sensitivity and interference; (5) questions asking the participants to inhibit their response to a stimulus that was previously administered (go-no-go test) to assess their inhibitory control; and (6) questions assessing the involuntary behavior that is triggered by sensory stimulation (prehension behavior) to detect deficits in the environmental autonomy. Each subtest had a score of 0 to 3 , with a total possible score of 18; a higher score indicated better function [19].

The results of the CNT were analyzed to find the correlation between the FAB test and neuropsychological evaluation for frontal lobe function. The CNT can evalu- ate most cognitive functions comprehensively, including the frontal lobe dysfunction, and detect mild cognitive deficits in brain disease [20-24]. The test method has been standardized [25], and the assessments are reliable and valid compared with other neuropsychological testing measures $[26,27]$. In addition, the CNT provides consistency and the convenience of administration and reduces errors during scoring and interpretation $[20,27]$. The Computerized Neurocognitive Function Test software version 4.0, which comprised of 17 computerized neurocognitive function tests, was used. The authors [2830] conducted a study to develop a computerized cognitive function assessment test; they verified the reliability and validity of the test and documented the standardized data of Korean adults.

The CNT consisted of four domains: (1) digit span and verbal learning tests for assessing memory, (2) visual and auditory continuous performance test and wordcolor test for assessing attention, (3) card-sorting test for assessing executive function, and (4) trail-making test for visuomotor coordination. During the digit span test, after viewing the number of sequences, the subjects click digits in a forward and backward sequence at a rate of one digit per second. During the verbal learning test, the subjects hear 15 target words five times and recall them immediately. After 20 minutes, the subjects recall the target words and then recall those words again after viewing another 15 words. In the visual and auditory continuous performance test, after viewing or hearing a number, the subjects click a mouse when the visual or auditory number is displayed on the screen, but not when other numbers appear. During this test, the correct response, reaction times, and commission errors are checked. In the word-color test, 24 cards showa mismatch between the name and color (green, blue, yellow, and red), and the subjects are asked to read as soon as possible. The subjects are then asked to read black letters of a color name (part A), color name (part B), name matching the correct color (part C), and color mismatching the correct name (part D). The response times are checked. With the card-sorting test for assessing executive function, there are six cards with different colors, shapes, and numbers. The subjects are asked to match the cards based on color, form, and number. The numbers of correctly matching cards are checked. Finally, the trail-making test for visuomotor coordination is performed. During part A of 
the trail-making test, the subjects are asked to draw a line with a computer mouse following sequential numbers. During part B of the trail-making test, sequential letters are added to the test. The time (in seconds) it took to complete the test is checked [21-23]. Because of the cost of the test, only the CNT data from subjects who agreed to undergo the test were collected. A well-trained therapist administered the FAB test and MMSE, and a skilled therapist, who was blinded to the results of the other cognitive function tests, administered the CNT.

\section{Statistical analysis}

All statistical analyses were performed using SPSS version 18.0 (SPSS Inc., Chicago, IL, USA). The Kruskal-Wallis test was used to compare the FAB and MMSE scores of the three groups. Considering the variables that affect the FAB score, an analysis of covariance (ANCOVA) was performed to determine if there were significant differences between the three groups regarding the stroke severity or age $[7,31]$. The stroke severity was evaluated using the initial NIHSS score.

The association of the FAB tests with the CNT and MMSE was analyzed using the Spearman correlation coefficient ( $\mathrm{r}$ ). In consideration that the FAB score is related to the MMSE score $[7,13,32]$, partial correlation analysis was performed to determine the correlation between the FAB test and CNT, excluding the effects of the MMSE. Statistical significance was set to $\mathrm{p}<0.05$.

\section{RESULTS}

Three hundred thirty-four patients who were admitted to the hospital for brain lesions from November 2016 to December 2017 underwent an assessment of their cognitive function. Of these, 18 patients with traumatic brain injury, 12 with brain tumor, 2 with Parkinson disease, 1 with Alzheimer disease, 2 with hypoxic brain injury, 8 without an initial NIHSS evaluation, and 37 with a history of stroke were excluded. Two hundred fifty-three stroke patients whose cognitive functions were evaluated and met the inclusion criteria were analyzed.

The patients were classified into three groups according to the area of the lesions involved. Sixty-six patients had direct frontal lobe damage. Overall, 7, 6, 10, 39, and 4 patients had anterior communicating aneurysm rupture, frontal lobe intracranial hemorrhage, anterior cerebral artery infarction, middle cerebral artery infarction, and anterior and middle cerebral artery infarction, respectively. One hundred twenty-two patients had frontal subcortical circuit lesions. Eighty-eight cases were caused by

Table 1. Baseline characteristics of the patients in this study

\begin{tabular}{lccc}
\hline & $\begin{array}{c}\text { Lesions involving the } \\
\text { frontal lobe cortex (n=66) }\end{array}$ & $\begin{array}{c}\text { Lesions related to the frontal } \\
\text { subcortical circuit }(\mathbf{n}=\mathbf{1 2 2})\end{array}$ & $\begin{array}{c}\text { Other lesions } \\
(\mathbf{n}=\mathbf{6 5})\end{array}$ \\
\hline Age (yr) & $64.3 \pm 13.9$ & $62.4 \pm 14.4$ & $62.4 \pm 12.3$ \\
Sex & 40 & & 45 \\
Male & 26 & 51 & 20 \\
$\quad$ Female & $41.2 \pm 53.8$ & $34.6 \pm 59.1$ & $25.3 \pm 52.0$ \\
Mean interval between stroke and & & & \\
$\quad$ cognitive function testing (day) & $35.3 \pm 27.2$ & $43.6 \pm 27.7$ & $47.6 \pm 26.8$ \\
MBI & $9.1 \pm 7.0$ & $7.1 \pm 7.1$ & $4.8 \pm 4.7$ \\
NIHSS & & & 53 \\
Type of stroke & 53 & 88 & 12 \\
Infarction & 13 & 34 & 30 \\
Hemorrhage & & & 27 \\
Location of stroke & 20 & 56 & 8 \\
Right & 37 & 62 & \\
Left & 9 & 4 & \\
$\quad$ Both & & & \\
\hline
\end{tabular}

Values are presented as mean \pm standard deviation.

MBI, Modified Barthel Index; NIHSS, National Institutes of Health Stroke Scale. 
an infarction, of which 36 were in the basal ganglia, 13 in the thalamus, 21 in the corona, and 18 with a combined lesion. Thirty-four cases were caused by hemorrhage, of which 24 in the basal ganglia, 8 in the thalamus, and 2 in the combined lesion. Sixty-five patients had other lesions that were not related to the frontal lobe circuit. The following conditions were encountered: 3 midbrain infarctions; 8 medulla infarctions; 5 cerebellum infarctions; 8 combined infarctions; 11 other cortex infarctions; 6 temporal, parietal, and occipital cortex hemorrhages; 3 intraventricular hemorrhages; and 3 subarachnoid hemorrhages. Table 1 lists the baseline characteristics of these patients.

The mean FAB scores were significantly different in the FL (7.3 \pm 5.0$)$, SC (9.5 \pm 5.3$)$, and OL groups (10.5 \pm 4.8$)$. The patients in the FL group had significantly lower total scores and FAB subtest scores (similarities, lexical fluency, motor series, conflicting instruction, go-no-go, and prehension behavior subtests) than those in the SC and
OL groups. The mean total FAB scores and subtest scores were significantly lower in the SC group than in the $\mathrm{OL}$ group (Table 2, Fig. 1).

Patients in the FL group (16.94 \pm 9.04$)$ had significantly lower mean MMSE scores than those in the SC $(20.68 \pm 8.38)$ and OL groups $(23.36 \pm 6.67)$. With the MMSE, the results of the subtest that were related to the frontal lobe function were analyzed further, and the patients in the FL groups exhibited significantly lower scores than those with the other lesions for the attention and calculation subtests and three-stage command subtest (Table 2, Fig. 1).

After controlling for the stroke severity using the initial NIHSS score, patients in the FL group had a significantly lower mean FAB score than those in the SC and OL groups. In the FAB subtests, the NIHSS-adjusted mean score and motor series, go-no-go, and prehension behavior subtests scores were significantly lower in the FL group compared with the other groups. When controlling

Table 2. Total and subtest scores of the FAB and MMSE of stroke patients

\begin{tabular}{|c|c|c|c|}
\hline & $\begin{array}{l}\text { Lesions involving the } \\
\text { frontal lobe cortex }\end{array}$ & $\begin{array}{l}\text { Lesions related to the } \\
\text { frontal subcortical circuit }\end{array}$ & Other lesions \\
\hline \multicolumn{4}{|l|}{ FAB score } \\
\hline Total $^{\text {a) }}$ & $7.28 \pm 4.99^{\mathrm{b}, \mathrm{e}}$ & $9.53 \pm 5.31$ & $10.48 \pm 4.83$ \\
\hline Similarities $^{\mathrm{a})}$ & $0.95 \pm 1.08^{\mathrm{f})}$ & $1.27 \pm 1.03$ & $1.37 \pm 1.00$ \\
\hline Lexical fluency $^{\text {a) }}$ & $0.45 \pm 0.77^{\mathrm{f})}$ & $0.72 \pm 0.85$ & $0.79 \pm 0.87$ \\
\hline Motor series $^{\mathrm{a})}$ & $\left.1.23 \pm 1.29^{\mathrm{b}, \mathrm{e}}\right)$ & $1.73 \pm 1.26$ & $1.88 \pm 1.22$ \\
\hline Conflicting instructions $s^{\text {a) }}$ & $1.19 \pm 1.17^{\mathrm{c}, \mathrm{e})}$ & $1.71 \pm 1.25$ & $1.90 \pm 1.28$ \\
\hline Go-no-go ${ }^{\text {a) }}$ & $1.05 \pm 1.09^{\mathrm{b}, \mathrm{e}}$ & $1.48 \pm 1.19$ & $1.64 \pm 1.15$ \\
\hline Prehension behaviour ${ }^{\mathrm{a})}$ & $2.36 \pm 1.14^{\mathrm{b}, \mathrm{f})}$ & $2.63 \pm 0.93$ & $2.89 \pm 0.52$ \\
\hline \multicolumn{4}{|l|}{ MMSE } \\
\hline Total $^{\mathrm{a})}$ & $\left.16.94 \pm 9.04^{\mathrm{b}, \mathrm{e}}\right)$ & $20.68 \pm 8.38$ & $22.29 \pm 7.92$ \\
\hline Three-stage command ${ }^{\mathrm{a})}$ & $1.96 \pm 1.06^{\mathrm{d}, \mathrm{f})}$ & $2.32 \pm 0.95$ & $2.32 \pm 0.97$ \\
\hline Attention and calculation ${ }^{\text {a) }}$ & $1.66 \pm 1.87^{\mathrm{b}, \mathrm{e})}$ & $2.36 \pm 1.93$ & $2.89 \pm 1.98$ \\
\hline Language (repetition) & $0.70 \pm 0.46$ & $0.81 \pm 0.41$ & $0.81 \pm 0.40$ \\
\hline
\end{tabular}

Values are presented as mean \pm standard deviation.

FAB, Frontal Assessment Battery; MMSE, Mini-Mental State Examination; NIHSS, National Institutes of Health Stroke Scale; FL group, with lesions involving the frontal lobe cortex; SC group, with lesions related to the frontal subcortical circuit without frontal cortex involvement; OL group, with other lesions that were unrelated to the frontal subcortical circuit.

${ }^{\text {a) }} \mathrm{p}<0.05$ indicate a significant difference among three groups.

${ }^{b} \mathrm{p}<0.05$ indicate a significantly different compared to SC and OL groups after controlling initial NIHSS score.

${ }^{c)} \mathrm{p}<0.05$ indicate a significantly different compared to OL group after controlling initial NIHSS score.

${ }^{d)} \mathrm{p}<0.05$ indicate a significantly different compared to SC group after controlling initial NIHSS score.

${ }^{\text {e) }} \mathrm{p}<0.05$ indicate a significantly different compared to SC and OL groups after controlling age.

${ }^{\mathrm{f}} \mathrm{p}<0.05$ indicate a significantly different compared to OL group after controlling age. 

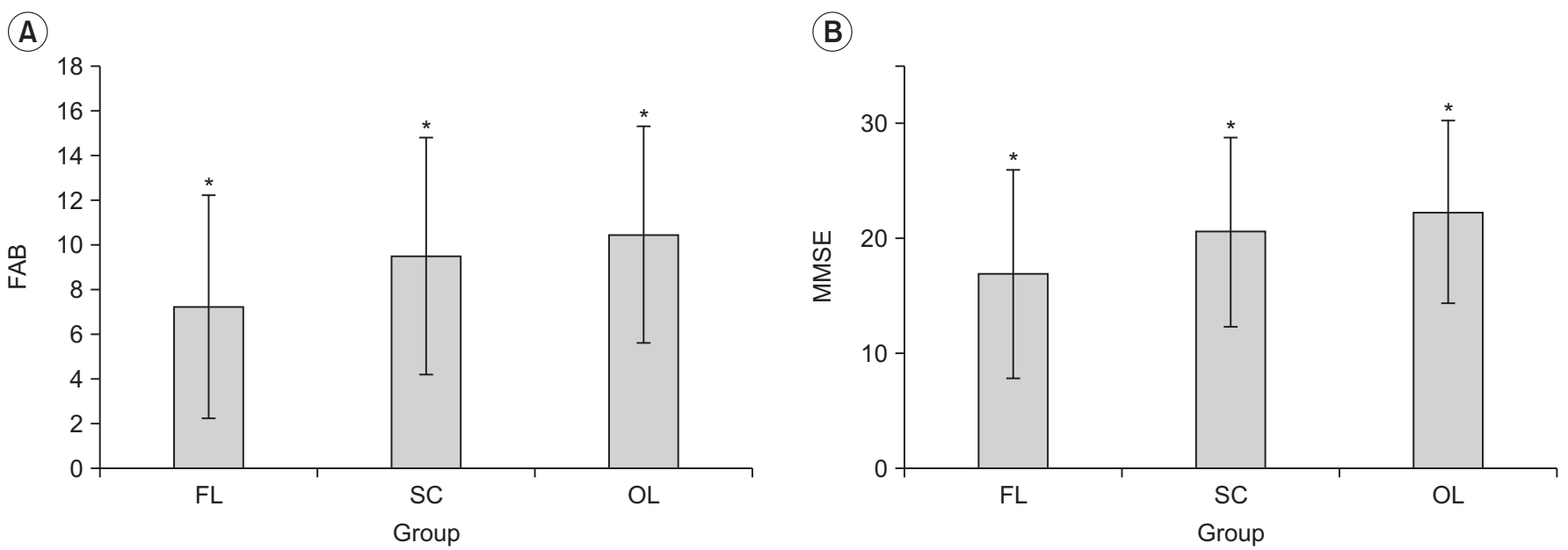

Fig. 1. Frontal Assessment Battery (FAB) and Mini-Mental State Examination (MMSE) scores of patients according to brain lesions. (A) The mean FAB scores were significantly different in the FL group (7.3 \pm 5.0$)$, SC group (9.5 \pm 5.3 ), and OL group (10.5 \pm 4.8$)$. (B) The mean MMSE scores were significantly different in the FL group (16.94 \pm 9.04$)$, SC group (20.68 \pm 8.38$)$, and OL group (23.36 \pm 6.67$)$. FL group, with lesions involving the frontal lobe cortex; SC group, with lesions related to the frontal subcortical circuit without frontal cortex involvement; OL group, with other lesions that were unrelated to the frontal subcortical circuit. ${ }^{*} \mathrm{p}<0.05$ indicate a significant difference among three groups.

for the age effect, patients in the FL group had significantly lower mean FAB scores and lower scores for the motor series, conflicting instructions, and go-no-go subtests than those in the SC and OL groups. The mean total MMSE score of the FL group was significantly lower than the other groups after controlling for the NIHSS score and age. On the other hand, the language subtest of the MMSE showed no significant difference among the three groups (Table 2).

After dividing the subjects into the FL, SC, and OL groups, the cutoff scores of the FAB test and MMSE were calculated to detect the frontal subcortical pathway lesions. A cutoff of 9.5 for the FAB test showed $60 \%$ sensitivity and $24 \%$ specificity, and a cutoff of 24.5 for the MMSE showed $38 \%$ sensitivity and $44 \%$ specificity. Inthe present study, the FAB and MMSE scores for detecting frontal subcortical circuit lesions showed poor accuracy.

Patients who agreed to undergo and completed the subtests of the CNT were analyzed. The following patients were investigated to determine the correlation between the FAB and MMSE scores: 96 and 56 patients who participated in trail-making A and trail-making B, respectively; and 166, 166, 163, 164, and 126 patients who participated in the card-sorting test, digital span tests, verbal learning test, visual and auditory continuous performance task (CPT), and the word color tests, respectively.

The total FAB score correlated with the tests of visuo- motor coordination (trail-making tests A and B), high cognitive function test (card-sorting test), memory tests (digital span and verbal learning), and attention tests (visual and auditory continuous performance tasks and word-color test). The MMSE score also correlated significantly with the scores of the trail-making A, card-sorting, and memory and attention tests. In contrast, trail-making B was not significantly correlated with the MMSE score. Even after controlling for the MMSE scores, the FAB scores were correlated with the trail-making A and B tests, card-sorting test, digital span tests, verbal learning test, and visual and auditory CPT (Table 3).

Scatterplots revealed a positive correlation and a significantly high correlation coefficient between the FAB test and MMSE. The high correlation coefficient between the FAB test and MMSE was sustained even after dividing among the three lesion groups (Fig. 2). Compared with the MMSE, the FAB test did not appear to detect the frontal lobe lesions in all the patients. In consideration that the frontal lobe function is composed of high order cognitive capacity and that the MMSE is not sensitive to mild cognitive impairment, the value of the FAB test was evaluated further according to the cognitive function level $[7,33,34]$. The patients were divided into high and low MMSE groups based on the MMSE cutoff score, meaning cognitively normal (MMSE=24) [35]. In the high MMSE group, there were 53, 30, and 83 patients in the FL, 
Table 3. Correlation between the CNT and total scores of the FAB and MMSE in patients with brain lesions

\begin{tabular}{|c|c|c|c|c|}
\hline Test & $\begin{array}{c}\text { Number of } \\
\text { patients }\end{array}$ & $\begin{array}{c}\text { Total FAB } \\
\text { score }\end{array}$ & $\begin{array}{l}\text { Total MMSE } \\
\quad \text { score }\end{array}$ & $\begin{array}{c}\text { Total FAB score with } \\
\text { a partial correlation after } \\
\text { controlling for the MMSE score }\end{array}$ \\
\hline \multicolumn{5}{|l|}{ Visuo-motor coordination } \\
\hline Trail making test $\mathrm{A}$ & 96 & $-0.467^{\mathrm{a})}$ & $-0.314^{\text {a) }}$ & $-0.405^{\mathrm{a})}$ \\
\hline Trail making test $\mathrm{B}$ & 56 & $-0.382^{\mathrm{b})}$ & -0.223 & $-0.394^{\text {a) }}$ \\
\hline \multicolumn{5}{|c|}{ High cognition function (executive function) test } \\
\hline Card sorting test & 166 & $0.645^{\mathrm{a})}$ & $0.606^{\mathrm{a})}$ & $0.273^{\mathrm{a})}$ \\
\hline \multicolumn{5}{|l|}{ Memory test } \\
\hline Digital span (forward) & 166 & $0.742^{\mathrm{a})}$ & $0.711^{\mathrm{a})}$ & $0.697^{\mathrm{a})}$ \\
\hline Digital span (backward) & 166 & $0.790^{\mathrm{a})}$ & $0.765^{\mathrm{a})}$ & $0.362^{\mathrm{a})}$ \\
\hline Verbal learning test (recall) & 163 & $0.702^{\mathrm{a})}$ & $0.687^{\mathrm{a})}$ & $0.690^{\mathrm{a})}$ \\
\hline Verbal learning test (interference) & 163 & $0.691^{a)}$ & $0.703^{\mathrm{a})}$ & $0.684^{\mathrm{a})}$ \\
\hline Verbal learning test (recognition) & 163 & $0.634^{\mathrm{a})}$ & $0.711^{\mathrm{a})}$ & $0.613^{\mathrm{a})}$ \\
\hline \multicolumn{5}{|l|}{ Attention test } \\
\hline Visual CPT (correct response) & 164 & $0.563^{\mathrm{a})}$ & $0.499^{\mathrm{a})}$ & $0.576^{\mathrm{a})}$ \\
\hline Visual CPT (commission response) & 164 & $-0.586^{a)}$ & $-0.521^{a)}$ & $-0.576^{\text {a) }}$ \\
\hline Visual CPT (reaction time) & 164 & $-0.199^{b)}$ & $-0.209^{a)}$ & $-0.216^{\text {a) }}$ \\
\hline Auditory CPT (correct response) & 164 & $0.645^{\mathrm{a})}$ & $0.606^{a)}$ & $0.612^{\mathrm{a})}$ \\
\hline Auditory CPT (commission response) & 164 & $-0.646^{\mathrm{a})}$ & $-0.609^{a)}$ & $-0.613^{\text {a) }}$ \\
\hline Auditory CPT (reaction time) & 164 & -0.046 & -0.017 & $-0.283^{a)}$ \\
\hline Word-Color test A & 126 & $-0.730^{\mathrm{a})}$ & $-0.598^{\mathrm{a})}$ & $-0.410^{\mathrm{a})}$ \\
\hline Word-Color test B & 126 & $-0.637^{\mathrm{a})}$ & $-0.586^{a)}$ & $-0.315^{\mathrm{a})}$ \\
\hline Word-Color test C & 126 & $-0.728^{a)}$ & $-0.664^{\text {a) }}$ & -0.001 \\
\hline Word-Color test D & 126 & -0.687 & $-0.602^{\text {a) }}$ & $-0.356^{\text {a) }}$ \\
\hline
\end{tabular}

CNT, Computerized Neuropsychological Function Test; FAB, Frontal Assessment Battery; MMSE, Mini-Mental State Examination; CPT, Continuous Performance Task.

${ }^{a)} \mathrm{p}<0.01,{ }^{\text {b) }} \mathrm{p}<0.05$ (Spearman correlation coefficients).

SC, and OL groups, respectively; 70, 82, and 31 patients in the FL, SC, and OL groups, respectively, belonged to the low MMSE group. The correlation coefficients between the FAB test and MMSE in the two groups were analyzed. A high MMSE score $(r=0.435, p<0.001)$ indicated a lower correlation with the FAB test score than a low MMSE score $(\mathrm{r}=0.714, \mathrm{p}<0.001)$.

In both the low and high MMSE groups, the total FAB score was correlated significantly with the tests of high cognitive function test (card-sorting test), memory tests (digital span and verbal learning test), and attention tests (visual and auditory continuous performance tasks). On the other hand, visuomotor coordination (trail-making tests A and B) test and attention (word card test D) meaningfully correlated with the total FAB score only in the high MMSE group (Table 4).

\section{DISCUSSION}

In this study, patients with frontal lobe lesions had significantly lower FAB total and subtest scores than those with the other types of lesions. In consideration of the initial stroke severity, the NIHSS-adjusted mean total FAB score and motor series, conflicting instructions, and go-no-go subtest scores of the FL group were significantly lower than those of the SC and OL groups. Forthe CNT, the FAB scores correlated with the measures of the visuomotor coordination, high cognitive function, and attention tests. Even after controlling for the MMSE scores, the FAB scores were correlated with all measures of the frontal lobe function. The trail-making B scores showed a significant correlation only with the FAB scores.

A positive correlation was observed between the MMSE 


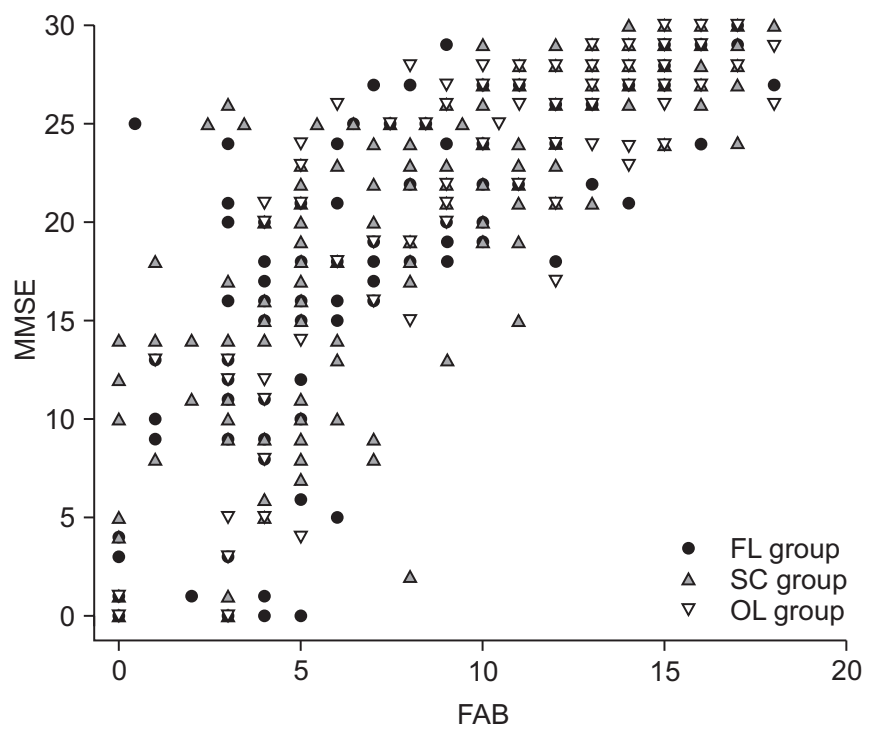

Fig. 2. Scatterplots for the Frontal Assessment Battery (FAB) and Mini-Mental State Examination (MMSE) scores of patients according to brain lesions. Scatterplots showed a positive correlation between the FAB and MMSE tests when divided among the three lesion groups (FL group: $\mathrm{r}=0.839, \mathrm{p}<0.001$; $\mathrm{SC}$ group: $\mathrm{r}=0.864, \mathrm{p}<0.001$; OL group: $\mathrm{r}=0.765, \mathrm{p}<0.001)$. When the MMSE scores were divided according to the cutoff value that (MMSE=24), a high MMSE score $(\mathrm{r}=0.488, \mathrm{p}<0.001)$ had a lower correlation with the FAB score than did a low MMSE score $(r=0.704$, $\mathrm{p}<0.001)$. FL group, with lesions involving the frontal lobe cortex; SC group, with lesions related to the frontal subcortical circuit without frontal cortex involvement; OL group, with other lesions that were unrelated to the frontal subcortical circuit.

and FAB scores when divided among the three lesion groups. When the MMSE scores were divided into groups based on the cutoff score for normal, a high MMSE $(\geq 24)$ score indicated a lower correlation with the FAB score than a low MMSE score $(<24)$.

In terms of the anatomical region of the brain, the FAB score showed a significant correlation with the frontal lobe lesions. The mean FAB scores were significantly lower for the patients with frontal lobe cortex and frontal subcortical circuit lesions than for the patients with other lesions. The mean FAB score was lowest for those with lesions in the frontal lobe cortex. This result was similar to that of a previous study showing that the FAB score was sensitive to frontal lobe damage in patients with right hemisphere stroke, as analyzed by voxel-based lesionbehavior mapping [16]. In particular, in each subtest in this study, patients with frontal cortex lesions had significantly low scores for all categories. This result was similar to the results of a previous study reporting that the inhibitory, conceptualization, and mental flexibility scores of the FAB were related to lateral prefrontal lesions [16].

The frontal subcortical network is the area of the cortexcaudate-globus pallidus-thalamus cortex and is determined according to three principal circuits: the dorsolateral prefrontal cortex (DLPFC), anterior cingulate circuit, and orbitofrontal circuits (OFC) [1]. Executive dysfunction and impairment in working memory are related to lesions of the DLPFC. Abulia and apathy are related to lesions of the anterior cingulate circuit, and OFC lesions cause disinhibition and mood disorders. When applied to the FAB subtest, the conceptualization, lexical fluency, and motor series were more related to the DLPFC. The results of the conflicting instructions, go-no-go, and prehension behavior subtests were related to the anterior cingulate circuit and OFC. In this study, the anatomical lesions correlated with all the FAB subtests. In terms of the value of the FAB for frontal lobe dysfunction detection, the FAB test significantly differentiated anatomical lesions from other types of lesions. After dividing the subjects into the FL, SC, and OL groups, the cutoff score of FAB was calculated to detect frontal subcortical pathway lesions. Meanwhile, the single score could not be used to detect frontal subcortical circuit lesions. This result was attributed to the heterogeneity of the anatomical lesions in the patients and the effects of global cognitive dysfunction influencing the frontal lobe domain.

In terms of the anatomy of the brain, previous studies examined the clinical usefulness of the FAB test for patients with other pathogeneses. Alzheimer disease patients with low FAB scores showed significant hypoperfusion in the left middle frontal gyrus, which was analyzed by single-photon emission computed tomography. These patients showed hypometabolism in the prefrontal regions according to fluorodeoxyglucose positron emission tomography $[36,37]$. For patients with Parkinson disease, the FAB scores correlated with the value of the gray matter in the right dorsolateral prefrontal cortex, which was analyzed by voxel-based morphometry [38]. In addition, Nakamura-Palacios et al. [39] reported an association between the FAB scores and the volume of gray matter in the prefrontal cortex and rostral middle frontal gyrus by segmenting the brain structures in alcoholic subjects and 
Table 4. Correlation between the CNT and total scores of the FAB divided by MMSE score (MMSE=24) in patients with brain lesions

\begin{tabular}{lll}
\hline \multicolumn{1}{c}{ Test } & MMSE $<\mathbf{2 4}(\mathbf{n}=\mathbf{8 6})$ & MMSE $\geq \mathbf{2 4}(\mathbf{n}=\mathbf{8 3})$ \\
\hline Visuo-motor coordination & & $-0.523^{\mathrm{a})}$ \\
Trail making test A & -0.336 & $-0.329^{\mathrm{a})}$ \\
\hline Trail making test B & -0.453 & \\
High cognition function (executive function) test & & $0.402^{\mathrm{a})}$ \\
$\quad$ Card sorting test & $0.502^{\mathrm{a})}$ & \\
Memory test & & $0.610^{\mathrm{a})}$ \\
Digital span (forward) & $0.548^{\mathrm{a})}$ & $0.570^{\mathrm{a})}$ \\
Digital span (backward) & $0.683^{\mathrm{a})}$ & $0.370^{\mathrm{a})}$ \\
Verbal learning test (recall) & $0.626^{\mathrm{a})}$ & $0.367^{\mathrm{a})}$ \\
Verbal learning test (interference) & $0.574^{\mathrm{a})}$ & $0.305^{\mathrm{a})}$ \\
Verbal learning test (recognition) & $0.474^{\mathrm{a}}$ & \\
Attention test & & $0.515^{\mathrm{a})}$ \\
Visual CPT (correct response) & $0.396^{\mathrm{b})}$ & $-0.515^{\mathrm{a})}$ \\
Visual CPT (commission response) & $-0.396^{\mathrm{b})}$ & -0.068 \\
Visual CPT (reaction time) & -0.160 & $0.365^{\mathrm{a})}$ \\
Auditory CPT (correct response) & $0.430^{\mathrm{a})}$ & $-0.368^{\mathrm{a})}$ \\
Auditory CPT (commission response) & $-0.430^{\mathrm{a})}$ & -0.148 \\
Auditory CPT (reaction time) & -0.036 & $-0.611^{\mathrm{a})}$ \\
Word-Color test A & $-0.597^{\mathrm{a})}$ & $-0.458^{\mathrm{a})}$ \\
Word-Color test B & $-0.704^{\mathrm{a})}$ & $-0.569^{\mathrm{a})}$ \\
Word-Color test C & $-0.609^{\mathrm{a})}$ & $-0.482^{\mathrm{a})}$ \\
Word-Color test D & -0.228 & \\
\hline
\end{tabular}

CNT, Computerized Neuropsychological Function Test; FAB, Frontal Assessment Battery; MMSE, Mini-Mental State Examination; CPT, Continuous Performance Task.

${ }^{a)} \mathrm{p}<0.01,{ }^{\text {b) }} \mathrm{p}<0.05$ (Spearman correlation coefficients).

viewing them with MRI. Considering its high correlation with the results of the neuroimaging technique, the FAB test is a useful assessment tool for detecting frontal lobe lesions in patients with a range of diseases. Conversely, because there is little data on stroke patients, further studies of the correlation between the FAB score and anatomical lesions analyzed with neuroimaging will be needed.

Significant correlations were shown between the results of the FAB and CNT for frontal lobe dysfunction. In the current study, there were correlations between the FAB scores and those of the trail-making part A and B test, card-sorting test, digital span and verbal learning tests, visual and auditory continuous performance test, and word-color test even after controlling for the MMSE scores. These results are consistent with those of previous studies of patients with a range of brain lesions. In
Parkinson disease, the FAB scores were correlated with measures of the executive function, such as the Wisconsin card-sorting test and parts A and B of the trail-making test, and memory function, such as the word fluency test $[7,9,14]$. In addition, frontal lobe hypoperfusion patients with Moyamoya disease revealed lower scores in the trail-making test, verbal learning test, digit span test, and visual and auditory continuous performance tests [22]. Patients with frontal lobe brain injury showed significantly lower scores in verbal learning and word-color tests [21]. This result was consistent with the fact that the frontal lobe is associated with attention and judgment and short-term memory [1,3,21-23].

The MMSE is one of the most frequently used tools for evaluating the cognitive function, but it has limitations in testing the function of the frontal lobe, including the executive function and attention domains. Regarding the 
MMSE score, patients in the FL and SC groups had significantly lower mean MMSE scores than patients with other types of lesions. In the executive function categories of the MMSE, there were meaningful differences among the three groups regarding attention and calculation and the three-stage command subtests. On the other hand, the language and memory subtest scores were not different in those groups. Additionally, the MMSE score correlated significantly with the scores of the trail-making A, cardsorting, and memory and attention tests. In contrast to the findings of this study, Slachevsky et al. [12] reported that the FAB scores were significantly different between patients with frontotemporal dementia and those with Alzheimer disease; however, the MMSE score was similar. In the present study, most of the FL and SC groups showed general cognitive dysfunction, which allowed the MMSE score to correlate meaningfully with the anatomical lesions and CNT results.

In this study, scatterplots showed a positive correlation and a significantly high correlation coefficient between the FAB and MMSE scores. This positive correlation was documented in patients with a range of pathologies, including Parkinson disease, Alzheimer disease, and frontotemporal dementia $[13,15,32]$. The MMSE had limitations because it underestimates cognitive impairment. Therefore, in the present study, the correlation was evaluated further by dividing the MMSE cutoff score for normal cognition. A high MMSE score had a significantly lower correlation coefficient than a low MMSE score. Patients with general cognitive impairment had lower FAB and MMSE scores. In contrast, patients considered to have a normal cognitive function by the MMSE score had different FAB scores depending on the cognitive domains of each test. In the correlation with CNT, the visuomotor coordination (trail-making tests A and B) and memory (word-color test) test correlated with the total FAB score only in the high MMSE group. Frontal lobe dysfunction, particularly those with an attention shift and inhibition derived from trail-making test B and word-color test, could be detected by the FAB test in normal MMSE cognitive scored patients [40]. The FAB test had benefit when detecting frontal lobe dysfunction in those with high MMSE scores, and it could be used to screen for subtle high cognitive dysfunction that the MMSE cannot detect.

This study had several limitations. First, the data were retrospectively reviewed using the medical records. Sec- ond, the number of patients was unevenly distributed among the anatomical lesion groups. Third, the interval between the stroke onset and assessment time could not be controlled for all patients. Conversely, almost all patients underwent cognitive function testing within 2 months of experiencing stroke, and none had chronic stroke more than 1 year after onset. Fourth, the stroke severity of the patients analyzed by the NIHSS was significantly different among the groups. Nevertheless, in consideration of the effects of the severity on the FAB and MMSE scores, this study analyzed the NIHSS-adjusted scores and found significant differences according to the anatomic brain lesions. Fifth, other high cognitive dysfunctions that affect the frontal lobe function, such as aphasia,were not considered. Finally, CNT version 4.0 was used for patients who were determined to require testing and agreed to pay for the test. Therefore, the results of CNT were limited to the subjects who agreed to undergo this test.

In conclusion, additional FAB testing could be used to detect frontal lobe dysfunction and quickly decide on treatment strategies for stroke patients, especially in normal MMSE cognitive scored patients.

\section{CONFLICT OF INTEREST}

No potential conflict of interest relevant to this article was reported.

\section{AUTHOR CONTRIBUTION}

Conceptualization: Han MH, Kim MW. Methodology: Han MH, Leigh JH. Formal analysis: Han MH, Kim DY. Visualization: Han MH. Writing - original draft: Han MH. Writing - review and editing: Han MH, Leigh JH, Kim MW. Approval of final manuscript: all authors.

\section{REFERENCES}

1. Hoffmann M. The human frontal lobes and frontal network systems: an evolutionary, clinical, and treatment perspective. ISRN Neurol 2013;2013:892459.

2. Stuss DT, Alexander MP. Executive functions and the frontal lobes: a conceptual view. Psychol Res 2000;63:289-98.

3. Estevez-Gonzalez A, Garcia-Sanchez C, Barraquer- 
Bordas L. Frontal lobes: the executive brain. Rev Neurol 2000;31:566-77.

4. Kokubo K, Suzuki K, Hattori N, Miyai I, Mori E. Executive dysfunction in patients with putaminal hemorrhage. J Stroke Cerebrovasc Dis 2015;24:1978-85.

5. Laplane D, Levasseur M, Pillon B, Dubois B, Baulac M, Mazoyer B, et al. Obsessive-compulsive and other behavioural changes with bilateral basal ganglia lesions: a neuropsychological, magnetic resonance imaging and positron tomography study. Brain 1989;112(Pt 3):699-725.

6. Bhatia KP, Marsden CD. The behavioural and motor consequences of focal lesions of the basal ganglia in man. Brain 1994;117(Pt 4):859-76.

7. Cohen OS, Vakil E, Tanne D, Molshatzki N, Nitsan $\mathrm{Z}$, Hassin-Baer S. The frontal assessment battery as a tool for evaluation of frontal lobe dysfunction in patients with Parkinson disease. J Geriatr Psychiatry Neurol 2012;25:71-7.

8. Zucchella C, Federico A, Martini A, Tinazzi M, Bartolo M, Tamburin S. Neuropsychological testing. Pract Neurol 2018;18:227-37.

9. Lima CF, Meireles LP, Fonseca R, Castro SL, Garrett C. The Frontal Assessment Battery (FAB) in Parkinson's disease and correlations with formal measures of executive functioning. J Neurol 2008;255:1756-61.

10. Takagi R, Kajimoto Y, Kamiyoshi S, Miwa H, Kondo T. The frontal assessment battery at bed side (FAB) in patients with Parkinson's disease. No To Shinkei 2002;54:897-902.

11. Biundo R, Weis L, Pilleri M, Facchini S, FormentoDojot P, Vallelunga A, et al. Diagnostic and screening power of neuropsychological testing in detecting mild cognitive impairment in Parkinson's disease. J Neural Transm (Vienna) 2013;120:627-33.

12. Slachevsky A, Villalpando JM, Sarazin M, Hahn-Barma V, Pillon B, Dubois B. Frontal assessment battery and differential diagnosis of frontotemporal dementia and Alzheimer disease. Arch Neurol 2004;61:1104-7.

13. Castiglioni S, Pelati O, Zuffi M, Somalvico F, Marino $\mathrm{L}$, Tentorio $\mathrm{T}$, et al. The frontal assessment battery does not differentiate frontotemporal dementia from Alzheimer's disease. Dement Geriatr Cogn Disord 2006;22:125-31.

14. Cunha PJ, Nicastri S, de Andrade AG, Bolla KI. The frontal assessment battery (FAB) reveals neurocogni- tive dysfunction in substance-dependent individuals in distinct executive domains: abstract reasoning, motor programming, and cognitive flexibility. Addict Behav 2010;35:875-81.

15. Paviour DC, Winterburn D, Simmonds S, Burgess G, Wilkinson L, Fox NC, et al. Can the frontal assessment battery (FAB) differentiate bradykinetic rigid syndromes? Relation of the FAB to formal neuropsychological testing. Neurocase 2005;11:274-82.

16. Kopp B, Rosser N, Tabeling S, Sturenburg HJ, de Haan B, Karnath HO, et al. Performance on the Frontal Assessment Battery is sensitive to frontal lobe damage in stroke patients. BMC Neurol 2013;13:179.

17. Fu C, Jin X, Chen B, Xue F, Niu H, Guo R, et al. Comparison of the Mini-Mental State Examination and Montreal Cognitive Assessment executive subtests in detecting post-stroke cognitive impairment. Geriatr Gerontol Int 2017;17:2329-35.

18. Trzepacz PT, Hochstetler H, Wang S, Walker B, Saykin AJ; Alzheimer's Disease Neuroimaging Initiative. Relationship between the Montreal Cognitive Assessment and Mini-Mental State Examination for assessment of mild cognitive impairment in older adults. BMC Geriatr 2015;15:107.

19. Dubois B, Slachevsky A, Litvan I, Pillon B. The FAB: a Frontal Assessment Battery at bedside. Neurology 2000;55:1621-6.

20. Gualtieri CT, Johnson LG. Reliability and validity of a computerized neurocognitive test battery, CNS Vital Signs. Arch Clin Neuropsychol 2006;21:623-43.

21. Kim YH, Shin SH, Park SH, Ko MH. Cognitive assessment for patient with brain injury by computerized neuropsychological test. J Korean Acad Rehabil Med 2001;25:209-16.

22. Kang CG, Chun MH, Kang JA, Do KH, Choi SJ. Neurocognitive dysfunction according to hypoperfusion territory in patients with moyamoya disease. Ann Rehabil Med 2017;41:1-8.

23. Cho HY, Kim KT, Jung JH. Effects of computer assisted cognitive rehabilitation on brain wave, memory and attention of stroke patients: a randomized control trial. J Phys Ther Sci 2015;27:1029-32.

24. Kim JY. Analysis of cognitive factors affecting stroke patient's activity of daily living performance: using the computerized neurocognitive function test. J Korea Acad Ind Coop Soc 2011;12:5715-21. 
25. de Oliveira MO, Brucki SMD. Computerized Neurocognitive Test (CNT) in mild cognitive impairment and Alzheimer's disease. Dement Neuropsychol 2014;8:112-6.

26. Mataix-Cols D, Bartres-Faz D. Is the use of the wooden and computerized versions of the Tower of Hanoi puzzle equivalent? Appl Neuropsychol 2002;9:117-20.

27. Parsey CM, Schmitter-Edgecombe M. Applications of technology in neuropsychological assessment. Clin Neuropsychol 2013;27:1328-61.

28. Kwon JS, Lyoo IK, Hong KS, Yeon BK, Ha KS. Development and standardization of the computerized memory assessment for Korean adults. J Korean Neuropsychiatr Assoc 2002;41:347-58.

29. Ha KS, Kwon JS, Lyoo IK, Kong SW, Lee DW, Youn T. Development and standardization process, and factor analysis of the computerized cognitive function test system for Korea adults. J Korean Neuropsychiatr Assoc 2002;41:551-62.

30. Lyoo IK, Kwon JS, Ha KS. Development and standardization of the computerized higher cortical function assessment for Korean adults J Korean Neuropsychiatr Assoc 2002;41:538-50.

31. Appollonio I, Leone M, Isella V, Piamarta F, Consoli T, Villa ML, et al. The Frontal Assessment Battery (FAB): normative values in an Italian population sample. Neurol Sci 2005;26:108-16.

32. Lipton AM, Ohman KA, Womack KB, Hynan LS, Ninman ET, Lacritz LH. Subscores of the FAB differentiate frontotemporal lobar degeneration from AD. Neurology 2005;65:726-31.

33. Dong Y, Venketasubramanian N, Chan BP, Sharma VK, Slavin MJ, Collinson SL, et al. Brief screening tests during acute admission in patients with mild stroke are predictive of vascular cognitive impairment 3-6 months after stroke. J Neurol Neurosurg Psychiatry 2012;83:580-5.

34. Nys GM, van Zandvoort MJ, de Kort PL, Jansen BP, Kappelle LJ, de Haan EH. Restrictions of the MiniMental State Examination in acute stroke. Arch Clin Neuropsychol 2005;20:623-9.

35. Sundar U, Adwani S. Post-stroke cognitive impairment at 3 months. Ann Indian Acad Neurol 2010;13:42-6.

36. Oshima E, Terada S, Sato S, Ikeda C, Nagao S, Takeda $\mathrm{N}$, et al. Frontal assessment battery and brain perfusion imaging in Alzheimer's disease. Int Psychogeriatr 2012;24:994-1001.

37. Lee JH, Byun MS, Sohn BK, Choe YM, Yi D, Han JY, et al. Functional neuroanatomical correlates of the frontal assessment battery performance in Alzheimer disease: a FDG-PET study. J Geriatr Psychiatry Neurol 2015;28:184-92.

38. Brugger F, Abela E, Hagele-Link S, Bohlhalter S, Galovic M, Kagi G. Do executive dysfunction and freezing of gait in Parkinson's disease share the same neuroanatomical correlates? J Neurol Sci 2015;356:184-7.

39. Nakamura-Palacios EM, Souza RS, Zago-Gomes MP, de Melo AM, Braga FS, Kubo TT, et al. Gray matter volume in left rostral middle frontal and left cerebellar cortices predicts frontal executive performance in alcoholic subjects. Alcohol Clin Exp Res 2014;38:112633.

40. Demakis GJ. Frontal lobe damage and tests of executive processing: a meta-analysis of the category test, stroop test, and trail-making test. J Clin Exp Neuropsychol 2004;26:441-50. 